\title{
Soziale Sicherung bei Arbeitslosigkeit - auf dem Weg in den Dualismus? Die Hartz-Reformen in historisch- komparativer Perspektive
}

Ein Jahrzehnt nach ihrer Umsetzung sind die Hartz-Reformen in der deutschen Öffentlichkeit noch immer stark umstritten. Vielfach ist zu hören, diese Reformen der staatlichen Arbeitslosenunterstützung hätten im Zusammenspiel mit Veränderungen auf dem Arbeitsmarkt neue Ungleichheiten hervorgebracht. In unserem Beitrag weisen wir dagegen nach, dass die Hartz-Reformen überkommene Differenzierungen hinsichtlich des Anspruchs auf Geldleistungen und der Gewährung von Wiedereingliederungsmaßnahmen in verschiedener Hinsicht sogar reduziert haben. Damit folgen sie einem Trend, der seit Ende der 1990er Jahre in verschiedenen europäischen Ländern zu beobachten ist.

JOCHEN CLASEN, DANIEL CLEGG

\section{Einleitung}

Es ist unstrittig, dass die Hartz-Reformen, insbesondere die seit 2005 umgesetzten Änderungen, tief in die Struktur des deutschen Arbeitslosensicherungssystems ${ }^{1}$ eingegriffen haben. Deutlich auseinander gehen die Meinungen aber, wenn es um die Charakterisierung dieser Reformen und ihre Bedeutung für das breitere Verständnis des deutschen Wirtschafts- und Sozialmodells sowie dessen Entwicklung geht. Wie von Bridgen und Meyer (2014) angemerkt, stehen sich bei der Betrachtung der Hartz-Reformen zwei Sichtweisen gegenüber. Auf der einen Seite wird argumentiert, die jüngsten Änderungen auf dem Gebiet der Arbeitslosensicherung seien als Teil und logische Folge eines breiteren wirtschaftlichen Deregulierungsprozesses zu sehen, der durch einen generellen Rückgang von Umfang und Tragweite staatlicher Intervention gekennzeichnet ist (Streeck 2009). Andere dagegen betrachten die Hartz-Reformen als Manifestation einer ganz speziellen Form oder Spielart von Liberalisierung, der sogenannten Dualisierung (Palier/Thelen 2012; Thelen 2012). Konkret bedeutet dies, dass die etablierten Sozialschutzmechanismen für Beschäftigte in den Kernsektoren der Wirtschaft - vor allem in der Fertigungsindustrie und in Dienstleistungssektoren mit hohen Qualifikationsanforderungen - erhalten oder sogar ausgebaut werden, während für die Arbeitnehmer in anderen Teilen des Arbeitsmarktes weniger großzügige, zweitklassige Regelungen gelten. Als emblematisch für diesen dualistischen Ansatz in der sozialen Sicherung, der institutionell zwischen generösen Leistungen für Insider und Regelungen am Existenzminimum für Outsider differenziert, wird die Einführung von Arbeitslosengeld I (ALG I) und Arbeitslosengeld II (ALG II) gesehen. Aus dieser Sicht fördern die jüngsten Reformen der Arbeitslosensicherung zudem ein kontinuierliches Auseinanderdriften des deutschen (bzw. kontinentaleuropäischen) Sozial- und Wirtschaftsmodells und der in anderen Industrieländern, speziell in Skandinavien und im englischsprachigen Raum, zu findenden Alternativen.

Indem wir die Hartz-Reformen in eine historischvergleichende europäische Perspektive setzen, hinterfragen wir im Folgenden ihre Darstellung als eine Verschiebung hin zu einem verstärkten Dualismus in der Arbeitslosensicherung. Wir stellen die Behauptung auf, dass sich überlappende institutionelle Differenzierungen - insbesondere zwischen Arbeitslosenversicherung und Arbeitslosenhilfe sowie zwischen Leistungen für Arbeitslose und für Nichterwerbstätige - bereits im sogenannten goldenen Zeitalter des Wohlfahrtsstaats nicht nur in

Den im Deutschen eher unüblichen Terminus „Arbeitslosensicherung" verwenden wir als Oberbegriff für Arbeitslosenversicherung und soziale Mindestsicherungen bei Arbeitslosigkeit, um umständlichere Umschreibungen zu vermeiden. 
Deutschland, sondern in allen Industrieländern konstitutive Merkmale gewesen sind und dass die Tragweite dieser Unterscheidungen infolge der Anstrengungen zur Konsolidierung der Staatshaushalte sowie der Steuerungsmaßnahmen für den Arbeitsmarkt in den Jahrzehnten nach den Ölkrisen Mitte der 1970er Jahre allerorten beträchtlich gestiegen ist. Weiterhin führen wir aus, dass seit Mitte der 1990er Jahre die Reformbewegung im Bereich der europäischen Arbeitslosensicherung, in die man auch die Hartz-Reformen einordnen kann, den Dualismus in mancher Hinsicht sogar abgeschwächt hat. Wenngleich dieser Beitrag der These von einer Dualisierung der Arbeitslosensicherung widerspricht, lautet das Fazit dennoch, dass die Änderungen, um die es hier geht, sich andererseits auch nicht einfach auf einen Liberalisierungsprozess reduzieren lassen - bestimmten Bevölkerungsgruppen haben sie eine bessere Absicherung beschert und staatliche Eingriffe zur Regelung des Arbeitslosigkeitsrisikos wurden nicht einfach zurückgeschraubt, sondern vom Ansatz her verändert.

\section{Die Hartz-Reformen: Dualisierung oder (eine andere Art von) Liberalisierung?}

Die Zusammenführung von Arbeitslosen und Sozialhilfe durch Hartz IV war der politisch umstrittenste und vielleicht augenfälligste Aspekt der Hartz-Reformen. Wenngleich in der Praxis eine große Mehrheit der Empfänger der vormaligen Arbeitslosenhilfe (ALH) aufgrund der Bedürftigkeitsprüfung nur in geringem Umfang Leistungen bezog, verkörperte die Abhängigkeit der Arbeitslosenhilfe vom früheren Verdienst symbolisch die Grundprinzipien, die der sozialen Sicherung zugrunde lagen - die Zugehörigkeit auch der Langzeitarbeitslosen zur Solidargemeinschaft und das Prinzip der Lebensstandarderhaltung. Die Abschaffung der Lohnproportionalität erweckte folglich den Eindruck, ein Teil der arbeitslosen Bevölkerung sollte von der allgemeinen Sozialsicherung abgekoppelt werden. Das aus dieser Reform entstandene zweigeteilte Leistungssystem für Arbeitslose („bifurcated benefits“, Hinrichs 2010) schien folglich eine neue scharfe Trennlinie zu ziehen zwischen denjenigen Arbeitslosen, die nach dem Verlust ihres Arbeitsplatzes weiterhin Anspruch auf verdienstabhängige Leistungen haben, und denjenigen, die infolge der Reform auf reine Sozialfürsorge angewiesen sind.

Vor diesem Hintergrund überrascht es nicht, dass viele Betrachter die Hartz-Reformen im Bereich der sozialen Sicherung Arbeitsloser als Ausdruck einer dualistischen Reformdynamik verstehen, welche sich durch „ungebrochene soziale Absicherung von Insidern und verstärkte Rekommodifizierung von Outsidern" auszeichnet (Fleckenstein 2012, S. 861). Aus institutioneller Sicht basiert eine dualistisch aufgebaute Arbeitslosensicherung auf einer Aufspaltung der Arbeitslosenunterstützung in zwei parallele Sicherungssysteme, welche die Erwerbslosen hinsichtlich ihres Anspruchs auf Hilfe zum Lebensunterhalt, ihrer Pflichten als Leistungsbezieher sowie des Umfangs und der Qualität ihnen zustehender Arbeitsmarktdienstleistungen sehr ungleich behandeln. Kernstück des angenommenen Dualismus ist eine wachsende Kluft zwischen Versicherungsleistungen und Sozialleistungen. Diese werden im Rahmen der Organisation von sozialer Sicherheit insgesamt nicht als komplementäre Instrumente, sondern als institutionell verschiedene, auf radikal unterschiedlichen Prinzipien und Zielsetzungen aufbauende Formen der Unterstützung gesehen, als zwei getrennte Wohlfahrtswelten innerhalb eines Sozialsystems (Palier 2010, S. 383).

Ein Trend hin zu einer zunehmend dualistischen Politik lässt sich in Untersuchungen zur langfristigen Anpassung der Arbeitslosensicherung an die Folgen der Ölkrise in den kontinentaleuropäischen Sozialsystemen konservativ-korporatistischer oder bismarckscher Prägung bereits seit längerer Zeit beobachten (Clasen/Clegg 2006; Clegg 2007). Die Arbeitslosenversicherung dieser Sozialsysteme, die auf den miteinander verwandten institutionellen Prinzipien der Finanzierung über parafiskalische Sozialabgaben und der autonomen korporatistischen Selbstverwaltung fußen, tendierte im Vergleich zu anderen Systemen stärker dazu, den ab den 1980er Jahren steigenden Kostendruck durch die Stärkung des Versicherungsprinzips aufzufangen. Dadurch wurden die - teilweise verbesserten - Leistungen der ersten Sicherungsstufe auf einen kleineren Empfängerkreis begrenzt. Ein Ergebnis dieses institutionell bedingten Musters politischer Anpassung war die Verlagerung von Langzeitarbeitslosen und Arbeitslosen mit kürzeren oder lückenhaften Beschäftigungszeiten auf vom Steuerzahler finanzierte und von öffentlichen Trägern erbrachte Sozialhilfeleistungen. Die institutionellen Unterschiede zwischen diesen Leistungen und jenen der Arbeitslosenversicherung waren in Wohlfahrtsstaaten bismarckscher Prägung generell ausgeprägter als in anderen Sozialsystemen.

Unlängst wurde eine weitere Betrachtungsweise der Dualisierung des Arbeitslosenschutzes vorgeschlagen, die vor allem auf politökonomischen Überlegungen aufbaut. Sie geht von der „suggestiven Parallele“ (Clegg 2007, S. 78) zwischen der Entstehung zweigeteilter Systeme zur Arbeitsmarktregulierung und der dualistischen Entwicklung der Arbeitslosensicherung in Kontinentaleuropa aus. Dabei betrachtet sie den Dualismus im Arbeitslosenschutz als Teil einer generellen Neuordnung der angenommenen institutionellen Komplementaritäten zwischen den industriellen Beziehungen und der Sozial- und Arbeitsmarktpolitik. Diese Neuorientierung ist durch die Herausforderungen des steigenden internationalen Wettbewerbs bedingt, mit dem sich die „koordinierten Marktwirtschaften“ konfrontiert sehen (Palier/Thelen 2012). Der Fortbestand großzügiger, lohnproportionaler Leistungen auf Basis der Arbeitslosenversicherung für Arbeitsmarkt-Insider ist nach dieser 
Interpretation notwendig, um die verstärkte Kooperation zwischen Kapital und Arbeitskräften in den Kernsektoren der Fertigungsindustrie und den Dienstleistungsbereichen mit hohen Qualifikationsanforderungen zu stützen. Dabei zielt die Zusammenarbeit der Sozialpartner im Wesentlichen darauf ab, die Produktivität in entscheidenden Wirtschaftsbereichen durch mehr interne Flexibilität zu steigern. Mit der Einführung eines nachgeordneten Absicherungsniveaus auf der Basis von Sozialhilfeleistungen und neuen, „atypischen“ Beschäftigungsverhältnissen segnet der Staat seinerseits das Entstehen eines parallelen, von hoher externer Flexibilität gekennzeichneten Arbeitsmarktes nicht nur $\mathrm{ab}$, sondern fördert es aktiv. Wirtschaftsaktivitäten mit geringen Qualifikationsanforderungen und niedrigem Produktivitätsniveau werden zunehmend auf diesen zweiten Arbeitsmarkt verlagert. So gesehen stellt die dualistische Arbeitslosensicherung nicht einfach eine rein reaktive und pfadabhängige Anpassung bestimmter Typen historisch gewachsener Leistungssysteme an den steigenden Kostendruck dar, sondern ist Teil eines "neuen Gleichgewichts“ (ebd., S. 218) im Verhältnis zwischen sozialer Sicherung und Produktion des Nationaleinkommens.

Diese zweite Erklärung für das Aufkommen des Dualismus beim Arbeitslosenschutz betrachtet den Sachverhalt aus dem Blickwinkel des Spielarten-des-Kapitalismus-Ansatzes ebenfalls als eine Entwicklung, die ausschließlich für bestimmte politökonomische Systeme typisch ist. In einem kürzlich erschienenen Beitrag systematisiert Thelen (2012) diese Argumentation und beschreibt drei Spielarten der Liberalisierung und die jeweils damit verbundenen Reformen der Arbeitslosensicherung (sowie andere Arten nichtmarktwirtschaftlicher Wirtschaftskoordination). Liberalisierung in Form von Dualisierung stellt aus dieser Perspektive ein Merkmal kontinentaleuropäischer Länder wie Deutschland oder Frankreich dar, wo die Verbandspolitik von einer Koalition großer Industriearbeitgeber und -gewerkschaften beherrscht wird, die lediglich einen geringen und zudem weiter schrumpfenden Anteil der Arbeitnehmerschaft vertreten, der sich überwiegend aus älteren Beschäftigten und vor allem aus männlichen Produktionsmitarbeitern zusammensetzt. In den koordinierten Marktwirtschaften Skandinaviens, deren Gewerkschaften über eine ungleich breitere Mitgliederbasis verfügen und wo das makrokorporatistische Erbe noch gegenwärtig ist, hat nach Thelen der Liberalisierungsprozess die Gestalt einer gesellschaftlich verankerten Flexibilisierung (socially embedded flexibilisation) angenommen. Der Arbeitslosenschutz sei hier im Wesentlichen unangetastet geblieben und werde von Maßnahmen aktiver Arbeitsmarktpolitik flankiert, die - dem viel gerühmten Flexicurity-Modell entsprechend den allgemeinen Kompetenzerwerb und die Arbeitskräftemobilität fördern. Im angelsächsischen Raum schließlich fand die Liberalisierung im Großen und Ganzen in Gestalt einer unverbrämten Deregulierung statt: Die Sozialleistungen wurden auf ein absolutes Minimum zurückgeschraubt, die Beschäftigungslosen an den Arbeitsmarkt verwiesen.
Die Erklärung der Hartz-Reformen als Teil eines Dualisierungsprozesses porträtiert diese Gesetzesänderungen als eine ganz spezielle Art von Arbeitslosensicherungsreform - möglicherweise vergleichbar mit Entwicklungen in anderen kontinentaleuropäischen Ländern, aber eindeutig zu unterscheiden von Reformen desselben politischen Themenfelds im skandinavischen oder englischsprachigen Raum. Dieser Darstellung zufolge haben die Hartz-Reformen eine, besonders die soziale Ungleichheit verschärfende Variante der Arbeitslosensicherung hervorgebracht. Ungleichheiten beim Absicherungsumfang sind demnach nicht das Ergebnis marktwirtschaftlicher Kräfte, sondern werden (zumindest in den koordinierten Marktwirtschaften) durch selektive und differenzierte Eingriffe staatlicher Politik institutionalisiert - ein Vorgang, der in der Dualisierungsthese als ein entscheidender Bruch mit dem Wohlfahrtsmodell des goldenen Zeitalters und seiner umfassenden, solidarischen Arbeitslosensicherung dargestellt wird.

\section{Differenzierte Arbeitslosensicherung während und jenseits des goldenen Zeitalters}

In der Praxis hingegen unterschied sich die Arbeitslosensicherung in den ersten Nachkriegsjahrzehnten durchaus von den Vorstellungen, die der Mythos vom goldenen Zeitalter heraufbeschwört. Im Widerspruch zu den Grundannahmen der Theorie von den Spielarten des Kapitalismus wiesen die entsprechenden Systeme in den meisten Industrieländern recht ähnliche Grundstrukturen auf und folgten einer vergleichbaren Logik. In Europa gab es zwar hinsichtlich der Finanzierung und Steuerung wichtige Unterschiede zwischen den einzelnen Ländern, aber auch unverkennbare, überall zu findende Gemeinsamkeiten.

Das Kernstück der sozialen Sicherung bei Arbeitslosigkeit war allerorten ein Versicherungssystem. Die Arbeitnehmer - zu jener Zeit überwiegend männliche Vollzeitbeschäftigte - leisteten während der Dauer ihrer Beschäftigung Beiträge, die ihnen im Fall von Arbeitslosigkeit, die damals hauptsächlich zyklischer Art war, zeitlich begrenzte, nicht bedarfsabhängige und meist lohnproportionale Geldleistungen sicherten. Wenngleich die Arbeitslosenversicherung vor dem Hintergrund einer generell positiven Nachfrage nach Arbeitskräften darauf abzielte, alle „normalen“ Arbeitslosigkeitsrisiken abzudecken, bezogen in den meisten Ländern bestimmte Beschäftigungslose dennoch Leistungen der allgemeinen Sozialhilfe. Diese wurden bedarfsabhängig gewährt, wenn die Voraussetzungen für den Bezug von Leistungen aus der Arbeitslosenversicherung nicht erfüllt waren, zum Beispiel aufgrund unzureichender Beitragszeiten oder, bei Langzeitarbeitslosen, weil der Anspruch erschöpft war. Zudem existierte in einigen Ländern - beispielsweise in 
Deutschland, Frankreich und den Niederlanden - ein gesondertes, von den Existenzminimums-Leistungen der Sozialhilfe getrenntes System der Arbeitslosenhilfe, dass weniger generös zahlte als die Arbeitslosenversicherung. In den Wohlfahrtsstaaten der Nachkriegszeit war die Arbeitslosensicherung überdies klar abgegrenzt von den - zusätzlich oder als Teil der Sozialhilfe gewährten - Unterstützungsleistungen für andere Personengruppen im Erwerbsalter, wie Menschen mit einer Behinderung, Langzeitkranke und später auch Alleinerziehende mit kleinen Kindern. Generell galten für die letztgenannten Unterstützungsleistungen andere, weniger strenge Anspruchs- und Bezugskriterien, da von diesem Empfängerkreis auch im Erwerbsalter nicht erwartet wurde, dass er bezahlte Arbeit verrichtete. Insgesamt lässt sich sagen, dass in den meisten Ländern Ungleichheiten hinsichtlich der Art und Höhe von Unterstützungsleistungen für Bevölkerungsgruppen mit unterschiedlicher Arbeitsbiografie ein Gründungsmerkmal der Systeme zur Arbeitslosensicherung in der Nachkriegszeit waren, auch wenn aufgrund der hohen Arbeitskräftenachfrage in den ersten Jahrzehnten nach dem Krieg den Sicherungsebenen unterhalb der Arbeitslosenversicherung kaum je größere Bedeutung zukam.

Mit dem Einsetzen von Massenarbeitslosigkeit und der Verlangsamung des Wirtschaftswachstums in den meisten Ländern Europas im Gefolge der Ölkrise der 1970er und 80er Jahre wandelte sich das Bild grundlegend. Unterschiedliche Reaktionen auf steigenden Problemdruck und schrumpfende Haushaltsmittel führten zu einer stärkeren Ausdifferenzierung der Systeme der Arbeitslosensicherung. In den skandinavischen Ländern, wie Dänemark, wurde der Zugang zu den Leistungen der Arbeitslosenversicherung erleichtert - de jure durch eine Verlängerung der Bezugszeiträume und de facto durch den Einsatz arbeitsmarktpolitischer Aktivierungsmaßnahmen zur Qualifizierung für den Bezug von Arbeitslosengeld -, um die Versorgung der wachsenden Zahl Langzeitarbeitsloser und junger Beschäftigungsloser sicherstellen zu können (Goul Andersen 2011). Im Vereinigten Königreich wurde ein entschiedener Sparkurs eingeschlagen. Der Anspruch auf Arbeitslosenversicherung wurde begrenzt, der Anteil der bedürftigkeitsgeprüften Leistungen an der Summe der Arbeitslosengeldzahlungen stieg dramatisch an und gewann bald das Übergewicht (Clasen 2011). In Ländern wie Frankreich und Deutschland nahmen die Kostensenkungsmaßnahmen während der 1980er und frühen 90er Jahre aus den oben angesprochenen Gründen vor allem Personengruppen am Rande des Arbeitsmarktes ins Visier - beispielsweise Berufseinsteiger, Gelegenheitsund Zeitarbeiter sowie die jüngeren Langzeitarbeitslosen. Diese Gruppen waren zunehmend auf Arbeitslosen- oder Sozialhilfe angewiesen (beziehungsweise auf damals neu eingeführte Regelungen wie in Frankreich die Arbeitslosenunterstützung (1984) und die Sozialunterstützung (1988)). Die Versicherungsleistungen für Personen, die länger oder mehr eingezahlt hatten, und insbesondere die für ältere $\mathrm{Ar}$ beitslose blieben dagegen weitgehend unangetastet oder erfuhren in Einzelfällen sogar eine Verbesserung (Clegg 2011;
Dingeldey 2011). Wenn überhaupt von einer Dualisierung der Arbeitslosensicherung in den bismarckschen Wohlfahrtsstaaten die Rede sein kann, dann war dies ihr Zeitalter.

Verbreiteter war unter den Ländern Europas eine zweite Reaktion auf die hohe Arbeitslosigkeit in den 1980er Jahren, und teilweise darüber hinaus, die den Bestrebungen nach Kosteneindämmung langfristig zuwiderlief. Gemeint ist die faktische Einschränkung des Schutzbereichs der Arbeitslosensicherung durch Verschiebung vieler Leistungsempfänger auf andere Arten der Unterstützung, die ohne Arbeitslosmeldung in Anspruch genommen werden konnten. Verschiedene Untersuchungen weisen nach, dass in den Ländern Europas im Laufe der 1980er Jahre unabhängig vom jeweiligen Wohlfahrtsmodell vielfältige Leistungsprogramme für Erwerbsfähige expandierten (siehe De Deken/Clasen 2013; Erlinghagen/Knuth 2010). Diese Strategie der Reduzierung des Arbeitskräfteangebots stützte sich auf den Ausbau bzw. die Einführung unterschiedlicher Vorruhestandsprogramme, zum Teil gefördert durch Reformen der Arbeitslosenversicherung. Zum Beispiel erhielten ältere Langzeitarbeitslose die Möglichkeit, in Rente zu gehen, oder der Bezugszeitraum der Arbeitslosenversicherung wurde für ältere Beschäftigungslose verlängert (Ebbinghaus 2006). Oder aber man verschob bestimmte Arbeitslose und Arbeitsuchende auf andere Leistungsprogramme, die sich eigentlich an Personenkreise im erwerbsfähigen Alter wandten, denen keine Beschäftigung zuzumuten war. Dies waren vor allem Programme für (geringfügig) Behinderte oder Langzeiterkrankte. Damit breitete sich eine Praxis aus, die in den Niederlanden schon seit den 1970er Jahren gang und gäbe gewesen war (Hoogenboom 2011, S. 76). Einige Länder führten neue Leistungen für bestimmte erwerbslose Altersgruppen ein (so beispielsweise 1976 in Frankreich die Beihilfe für Alleinerziehende) oder sie verliehen diesen Personenkreisen einen Sonderstatus im Rahmen der Sozialhilfe (z. B. Alleinerziehende im Vereinigten Königreich). Somit könnte man argumentieren, dass in jenen Jahren die Arbeitslosigkeit politisch und verwaltungstechnisch umkonstruiert und die dadurch vom Leistungsbezug Ausgeschlossenen auf sozialstaatliche Programme für Nichterwerbspersonen verwiesen wurden.

Zusammenfassend lässt sich sagen, dass im Zeitraum von Mitte der 1970er bis Mitte der 1990er Jahre, als sich Regierungen länderübergreifend mit den wirtschaftlichen und sozialen Folgen von Massenarbeitslosigkeit konfrontiert sahen, wichtige institutionelle Unterscheidungen - zwischen Arbeitslosenversicherung und Arbeitslosenhilfe, zwischen Leistungen für Beschäftigungslose und solchen für andere Personen im erwerbsfähigen Alter - an Bedeutung gewannen, die während der ausgedehnten Periode starken Wirtschaftswachstums und niedriger Arbeitslosigkeit in den ersten Nachkriegsjahrzehnten mehr oder weniger latent bereits existiert hatten. Es gab zwar gewisse Unterschiede zwischen den Ländern, vor allem in Bezug auf das Ausmaß von Restriktionen bei der Arbeitslosensicherung und die betroffenen Zielgruppen. Insgesamt allerdings wurden in diesem Zeitraum länderübergreifend die Rechte beschäftigungsloser Perso- 
nen zunehmend ausdifferenziert - nach Alter, persönlicher Erwerbsbiografie sowie in bestimmten Fällen nach Gesundheitszustand und familiärer Situation.

\section{Aktivierungsagenda und integrative Reformen der sozialen Sicherung bei Arbeitslosigkeit}

Etwa Mitte der 1990er Jahre begann eine neue Phase in der Geschichte der Arbeitslosensicherung in Europa. Ähnlich gelagerte institutionelle Veränderungen in einer Reihe europäischer Länder bewirkten eine grundlegende Neugestaltung der sozialen Sicherung bei Arbeitslosigkeit, vielfach unter Aufhebung der in den Vorjahren im Rahmen des Krisenmanagements durchgesetzten Reformen. Allerdings ist dieser Prozess nicht einfach auf eine Rückkehr zu dem Absicherungsmodell hinausgelaufen, das bis zu den 1970er Jahren in Europa überwogen hatte - viele der Reformen beseitigten aktiv die verwaltungstechnischen und politischen Unterscheidungen, die Merkmale des Paradigmas des goldenen Zeitalters gewesen waren. In diesem Sinne lassen sich diese Reformen als integrativ bezeichnen (Clasen/Clegg 2011). Wenngleich die Kostendämpfung weiterhin eine wichtige Rolle spielte, ist die Haupttriebfeder nun zum ersten Mal die sogenannte Aktivierungsagenda. Die primäre Rolle der Arbeitslosenabsicherung wird neu definiert und besteht nun darin, die Partizipation und Wiedereingliederung an bzw. in den Arbeitsmarkt zu fördern, und zwar nicht nur mit Blick auf die arbeitslos gemeldeten, sondern zunehmend auch auf andere Leistungsbezieher erwerbsfähigen Alters (Bonoli 2013).

Eine Dimension dieses Trends ließe sich als Homogenisierung der Arbeitslosensicherung beschreiben. Dies bedeutet, dass Sicherungsansprüche bei Arbeitslosigkeit, ausgedrückt in Leistungshöhe und Anspruchsdauer, weniger stark an persönliche Arbeitsbiografien und Beitragszeiten gekoppelt werden. Die Verknüpfung höherer einkommensabhängiger Leistungen mit langer Bezugsdauer an lange Beitragszeiten diente ursprünglich dem Ziel, stabile Beschäftigungsmuster im Arbeitsmarkt zu prämieren sowie für bestimmte Betroffene (und ältere Arbeitnehmer) Wege aus dem Arbeitsmarkt zu schaffen. Im Rahmen des Aktivierungsparadigmas verlieren diese Funktionen ihre Bedeutung. Die neue Rolle der Arbeitslosensicherung besteht darin, die Beschäftigungs- (und Lohn)flexibilität zu fördern und die Arbeitsmarktbeteiligung über das gesamte Altersspektrum hinweg zu maximieren. In dem Maße wie Arbeitslosenversicherungen ihre lebensstandarderhaltende Funktionen einbüßen, schwinden die Unterschiede hinsichtlich Leistungsanspruch und Bezugsdauer für Arbeitnehmer, die mit sehr unterschiedlicher beruflicher Vorgeschichte ihre Beschäftigung verlieren. Dies kann vielfach auch eine gewisse Angleichung der Rechte (und Pflichten) Arbeitsuchender zur Folge haben und damit zu einer allmählichen Erosion der Unterscheidung zwischen Versicherungs- und Fürsorgeleistungen führen. Eine andere Art der Homogenisierung würde bedeuten, dass unterschiedliche Leistungsstufen weiter bestehen, wobei allerdings entweder die Arbeitslosenversicherung (in weniger lohnbezogener Form) oder die Arbeitslosenhilfe (bzw. Sozialhilfe) bei der Sicherung von Arbeitslosigkeit dominiert (Homogenisierung nach oben bzw. nach unten).

Die Harz-Reformen sind ein Beispiel solch einer Homogenisierung nach unten. Die Verkürzung des Anspruchs auf Arbeitslosengeld für ältere Arbeitnehmer mit entsprechenden Beitragsleistungen hat die Arbeitsmarkt-Insider in einem Ausmaß getroffen, das nicht zur Dualisierungsthese passt. Die Reduzierung der Zahl separater Unterstützungsprogramme hat zu einem Übergewicht der bedarfsgeprüften Leistungen bei Arbeitslosigkeit geführt (Clasen/Goerne 2011). Ähnliche Entwicklungen lassen sich in Großbritannien (in den 1980er Jahren) beobachten wie auch in Dänemark, wo eine unzureichende Anpassung der absoluten Berechnungsobergrenze zu einer faktisch immer flacher werdenden Leistungsstruktur der Arbeitslosenversicherung geführt hat und die Höchstbezugsdauer im Zuge sukzessiver Reformen während der späten 1990er und frühen 2000er Jahre deutlich beschnitten wurde (Goul Andersen 2011).

Fälle einer reinen Anpassung nach oben lassen sich in der Praxis schwerer nachweisen. Der veränderte Grundansatz der Arbeitslosenversicherungsreform in Frankreich in den 2000er Jahren bietet Beispiele für den Versuch, die Arbeitslosensicherung inklusiver zu gestalten (Clegg 2011). Mit der Absicht, gewerkschaftliche Unterstützung für einen ehrgeizigen Aktivierungsansatz in der Arbeitslosenversicherung zu gewinnen, einigten sich die französischen Arbeitgeber und die Regierung 2001 auf eine beträchtliche Senkung der Mindestbeitragszeiten für den Bezug von Leistungen aus der Arbeitslosenversicherung sowie auf die Abschaffung der sogenannten Degressionsregelung, die die Entwicklung der Leistungshöhe an die geleisteten Beitragszahlungen knüpfte. Das Ergebnis war ein scharfer Anstieg der Zahl von Anspruchsberechtigten. 2009 handelten die Sozialpartner eine Reform aus, welche die Bezugsberechtigung für Arbeitnehmer mit sehr kurzen Beitragszeiten weiter verbesserte. Finanziert wurde diese Änderung durch eine Verkürzung der Dauer des Höchstanspruchs für Arbeitslose mit längeren Versicherungszeiten.

Eine zweite - und mit der ersten verbundene - Dimension integrativer Reformen in der Sozialpolitik ließe sich als Rekategorisierung bezeichnen. Das Paradigma des goldenen Zeitalters etablierte eine eindeutige Differenzierung von regulären Arbeitslosen und anderen nicht beschäftigten Personen im erwerbsfähigen Alter. In vielen Ländern schwoll der letztgenannte Personenkreis aufgrund der oben angesprochenen Politik in den 1980er und frühen 90er Jahren erheblich an. Für die Empfänger der jeweiligen Leistungen galten unterschiedliche Voraussetzungen sowohl hinsichtlich der Leistungshöhe 
als auch der Inanspruchnahme arbeitsmarktpolitischer Maßnahmen. Entsprechend der Zielsetzung, die Arbeitsmarktbeteiligung zu erhöhen, haben jüngste Reformen erneut die Kategorie Arbeitslosigkeit administrativ ausgeweitet. Dies geschah insbesondere durch (Wieder)Einbeziehung von Empfängern anderer - insbesondere bedürftigkeitsabhängiger Leistungen für Personen erwerbsfähigen Alters in die Arbeitslosensicherung oder durch stärkere Gleichstellung dieses Personenkreises mit Empfängern von Arbeitslosengeld, z. B. indem man das Erfordernis der aktiven Arbeitsuche auf sie ausdehnte. In Verbindung mit dem ersten Trend führt diese Entwicklung zur Zusammenfassung vormals separater Leistungsprogramme für Personen erwerbsfähigen Alters in einer einheitlichen, beschäftigungsorientierten Regelung, innerhalb derer die Ungleichheiten in der Behandlung von Leistungsempfängern lediglich von einer - wie auch immer gelagerten - Beurteilung ihrer Beschäftigungsfähigkeit abhängen. Programme dieser Art verschmelzen unter Umständen sogar die bedürftigkeitsabhängigen Leistungen für Arbeitslose mit (einkommensergänzenden) Unterstützungsleistungen für Beschäftigte. In diesem Sinne könnte man sagen, dass die vormalig eng umrissene Risikokategorie Arbeitslosigkeit durch die Ausdehnung der Kriterien Beschäftigungsfähigkeit und Arbeitsuche als Grundvoraussetzung für die Gewährung von sozialen Sicherungsleistungen erweitert, bzw. substituiert wurde.

Die Hartz-Reformen sind auch in dieser Hinsicht ein Beispiel für diesen Trend. Zwar wurde die Einführung von ALG II hauptsächlich vor dem Hintergrund der Verschiebung der Langzeitarbeitslosen in den Bereich der Fürsorge diskutiert, jedoch verschob die Reform auch erwerbsfähige Leistungsempfänger aus der von den Gemeinden getragenen Sozialhilfe in den Zuständigkeitsbereich der gesamtstaatlichen Arbeitslosensicherung und verschaffte ihnen damit Zugang zu verbesserten (und, da nicht mehr ortsabhängig, weniger differenzierten) Arbeitsförderungsleistungen (siehe Clasen/Goerne 2011; Dingeldey 2011, S. 63). In vielen Fällen dient das ALG II zudem zur Aufstockung des Einkommens aus einem Beschäftigungsverhältnis. In Frankreich brachte die Einführung des Revenue de Solidarité Active (RSA) im Jahr 2008 vergleichbare Änderungen, die vormals separaten kategorialen Beihilferegelungen für Nichterwerbstätige und unterstützungsbedürftige Erwerbstätige wurden in einem allgemeinen sozialen Sicherungssystem zusammengeführt. Um RSA-Leistungen beziehen zu können, müssen sich Arbeitslose nun ebenso wie bestimmte unterstützungsbedürftige Beschäftigte persönlich beim öffentlichen Arbeitsvermittlungsdienst melden. Nach den Regeln des früheren Sozialhilfesystems war dies nicht erforderlich (Clegg 2011, S. 45). In den Niederlanden erfolgte die Verschmelzung von Sozialhilfe und Arbeitslosenunterstützung bereits 1995. Seitdem unterlagen die meisten Leistungsempfänger Aktivierungsbedingungen. Im Zuge weiterer Reformen in den 2000er Jahren wurden verbleibende gruppenspezifische Ausnahmen von den Aktivierungsanforderungen (für Alleinerziehende und ältere Leistungsempfänger) aufgehoben. Zudem wurde die Finanzierung umgestellt, um die Gemeinden als Träger dazu anzuregen, die Aktivierung energischer voranzutreiben (Hoogenboom 2011, S. 87). Diese Art integrativen Wandels beschränkt sich jedoch nicht auf Kontinentaleuropa, sondern findet sich - möglicherweise in seiner radikalsten Ausprägung - ebenso in Großbritannien. Das System des Universal Credit, dessen Einführung 2013 begann, verschmilzt eine Reihe bisheriger bedürftigkeitsgeprüfter Leistungen für Arbeitslose und Nichterwerbstätige (in erster Linie Alleinerziehende und Behinderte) mit dem bisher separaten Leistungssystem für geringverdienende Erwerbstätige (Clasen 2011, S. 25).

Die Wende hin zur Aktivierungspolitik in den letzten Jahren hat also die Differenzierung innerhalb der Arbeitslosensicherungssysteme keineswegs verstärkt, sondern in mancher Hinsicht klar abgeschwächt. Die Veränderungen gingen mit umfassenden Reformen der Administration und Governance einher. In Deutschland, Frankreich und den Niederlanden beispielsweise wurde unter anderem die Bedeutung der Selbstverwaltung in der Arbeitslosenversicherung beschnitten oder gar aufgehoben; in Deutschland, aber auch in Dänemark, wurden die bisher geteilten, auf staatlicher bzw. lokaler Ebene angesiedelten Leistungssysteme für die verschiedenen Empfängergruppen durch neu geschaffene One-Stop-Shops ersetzt. Der integrative Denkansatz, der die Reform der Arbeitslosensicherung in Europa auszeichnet, ist somit nicht ausschließlich auf die Rechte und Pflichten verschiedener Gruppen von Leistungsempfängern im Erwerbsalter gerichtet, sondern auch auf die Organisationsstrukturen der sozialen Sicherung für diese Bevölkerungsgruppen (Champion/Bonoli 2011).

\section{Fazit}

Die Hartz-Reformen wurden vielfach mit dem im Trend liegenden Thema einer schleichenden Dualisierung der Arbeitsmarkt- und Sozialpolitik in Verbindung gebracht, was angesichts der Entstehung eines neuen Systems nominell zweigeteilter Leistungen im Zuge der Einführung von ALG I und ALG II sicher auch verständlich ist. Durch die Betrachtung dieser Reformen in historisch-komparativer Perspektive vertreten wir in diesem Beitrag das Argument, dass eine solche Charakterisierung nicht nur die Leitgedanken dieser grundlegenden Wende in der Arbeitsmarktpolitik verzerrt darstellt, sondern ebenso die Zielsetzungen einer europaweiten Welle von Reformen der Arbeitslosensicherung, die den Zeitraum von der Mitte der 1990er Jahre bis zum Eintritt der Wirtschaftskrise umspannt. Die interne Differenzierung der Anspruchsberechtigung ist ein seit langer Zeit bestehendes Merkmal der Sicherungssysteme für Arbeitslose, das mit dem Auftreten von Massenarbeitslosigkeit Mitte der 1970er bis Anfang der 90er Jahre beträchtlich an Bedeutung gewann. In jüngerer Zeit dagegen hat in verschiedenen Ländern eine Reihe integrativer Reformen zu einer verminderten 
Differenzierung der Rechte (und Pflichten) von Leistungsempfängern im erwerbstätigen Alter geführt. Obwohl die Unterschiede in der Arbeitslosensicherung nicht völlig beseitigt wurden - und es sicherlich auch nie werden -, war der jüngste Beobachtungszeitraum eher von einer Verwischung als von einer Verstärkung solcher Ungleichheiten gekennzeichnet.

Wenn die jüngsten Reformen in Deutschland und anderswo nicht dem Dualisierungsprinzip gefolgt sind, wie ließen sie sich dann am besten beschreiben? Zwar war die Aktivierungswende eindeutig darauf ausgerichtet, den Alternativen zur Teilnahme am Arbeitsmarkt einen Riegel vorzuschieben, und sie hat für manche Betroffene erhebliche Leistungskürzungen bedeutet - dennoch lässt sich nicht ohne Weiteres sagen, dass diese Veränderungen auf eine eindeutige Liberalisierung oder Deregulierung hinauslaufen. Für bestimmte Gruppen von Leistungsempfängern - in Deutschland viele der vormaligen Sozialhilfebezieher - dürften diese Änderungen die Qualität der sozialen Sicherung nicht verschlechtert und teilweise sogar verbessert haben, während die staatliche Intervention im Bereich der Arbeitslosensicherung nicht einfach zurückgefahren, sondern eher umgelenkt und umgestaltet wurde. Es hat folglich den Anschein, dass soziale Reformen länderübergreifend Elemente einer unverhüllten Liberalisierung mit dem verbänden, was Thelen (2012) als „embedded flexibilisation“ (gesellschaftlich eingebettete Flexibilisierung) bezeichnet, wenn auch die Kombination als solche von Fall zu Fall variiert. In der deutschen Arbeitslosensicherung geht die Entwicklung in Richtung eines Hybrids zwischen den politischen Ansätzen, die traditionell als angelsächsisches Modell (d.h. Leistungen in Höhe einer Grundsicherung) bzw. skandinavisches Modell (d.h. umfassende Aktivierung) bezeichnet werden.

Natürlich hat sich der Kontext der Arbeitslosensicherung seit dem Beginn der Wirtschaftskrise 2008 grundlegend geändert. In Anbetracht dessen dürfte die große Anzahl parametrischer Reformen der Sicherungssysteme für Arbeitslose, die quer durch Europa stattgefunden haben, nicht überraschen. Einige dieser Änderungen, wie die Ausweitung der Kurzarbeit, scheinen in einem Widerspruch zu den oben geschilderten integrativen Tendenzen zu stehen. Andere, wie die Senkung der Beitragsvoraussetzungen für den Bezug von Leistungen aus der Arbeitslosenversicherung in Italien, Vorschläge zur Begrenzung der maximalen Einkommensersatzraten in Frankreich oder die Weiterführung der Integration von Unterstützungsprogrammen für Erwerbstätige verschiedener Altersgruppen in den Niederlanden, erscheinen dagegen durchaus im Einklang mit der hier angeführten integrativen Tendenz zu sein. Angesichts des starken Anstiegs des Arbeitslosigkeitsrisikos für Kernbeschäftigte in manchen Ländern, gepaart mit einem hohen Druck auf die öffentlichen Haushalte, ist es eher überraschend, dass die institutionelle Entwicklung in der europäischen Arbeitslosensicherungspolitik ihren Kurs während der Krise nicht stärker geändert hat: zum Beispiel in Richtung auf einen ausgeprägteren Dualismus. Dies deutet darauf hin, dass die Integrationsdynamik, die die Reformen der Arbeitslosensicherung seit der Mitte der 1990er Jahre überwiegend auszeichnet, mittlerweile fest verankert scheint.

\section{LITERATUR}

Bonoli, G. (2013): The origins of active social policy, Oxford

Bridgen, P./Meyer, T. (2014): The liberalisation of the German social model: public-private pension reform in Germany since 2001, in: Journal of Social Policy 43 (1), S. $37-68$

Champion, C./Bonoli, G. (2011): Institutional fragmentation and coordination initiatives in western European welfare states, in: Journal of European Social Policy 21 (4), S. 323-334
Clasen, J. (2011): The United Kingdom: towards a single working-age benefit system, in: Clasen, J./Clegg, D. (Hrsg.), a. a. O., S. 15-33

Clasen, J./Clegg, D. (2006): Beyond activation. Reforming European employment protection systems in post-industrial labour markets, in: European Societies 8 (4), S. $527-553$

Clasen, J./Clegg, D. (Hrsg.) (2011): Regulating the risk of unemployment. National adaptations to post-industrial labour markets in Europe, Oxford

Clasen, J./Clegg, D. (2011): Unemployment protection and labour market change in Europe: towards 'triple integration'?, in: Clasen, J./Clegg, D. (Hrsg.), a. a. O., S. 1-12

Clasen, J./Goerne, A. (2011): Exit Bismarck, enter dualism? Assessing contemporary German labour market policy, in: Journal of Social Policy 40 (4), S. $795-810$

Clegg, D. (2007): Continental drift: on unemployment policy change in Bismarckian welfare states, in: Social Policy and Administration 41 (6), S. 597-617

Clegg, D. (2011): France: integration versus dualization, in: Clasen, J./Clegg, D. (Hrsg.), a. a. O., S. 34-54

De Deken, J./Clasen, J. (2013): Benefit dependency: the pros and cons of using 'caseload' data for national and international comparisons, in: International Social Security Review 66 (2), S. 53-78

Dingeldey, I. (2011): Germany: moving towards integration whilst maintaining segmentation, in: Clasen, J./Clegg, D. (Hrsg.), a. a. O., S. 55-74

Ebbinghaus, B. (2006): Reforming early retirement in Europe, Japan and the USA, Oxford

Erlinghagen, M./Knuth, M. (2010): Unemployment as an institutional construct? Structural differences in non-employment between selected European countries and the United States, in: Journal of Social Policy 39 (1), S. 71-94

Fleckenstein, T. (2012): The politics of labour market reform and social citizenship in Germany, in: West European Politics 35 (4), S. 847-868

Goul-Andersen, J. (2011): Denmark: ambiguous modernization of an inclusive unemployment protection system, in: Clasen, J./Clegg, D. (Hrsg.), a. a. O., S. $187-207$

Hinrichs, K. (2010): A social insurance state withers away: welfare reforms in Germany - or, attempts to turn around in a cul-de-sac, in: Palier, B. (Hrsg.): A long goodbye to Bismarck? The politics of welfare reform in continental Europe, Amsterdam, S. 45-72

Hoogenboom, M. (2011): The Netherlands: two tiers for all, in: Clasen, J./Clegg, D. (Hrsg.), a. a. O., S. 75-99

Palier, B. (2010): The long conservative corporatist road to welfare reforms, in: Palier, B. (Hrsg.): A long goodbye to Bismarck? The politics of welfare reform in continental Europe, Amsterdam, S. 333-388

Palier, B./Thelen, K. (2012): Dualization and institutional complementarities: industrial relations, labour market and welfare state changes in France and Germany, in: Emmenegger, P./Häusermann, S./Palier, B./Seeleib-Kaiser M. (Hrsg.): The age of dualization: the changing face of inequality in deindustrializing societies, New York, S. 201-225

Streeck, W. (2009): Re-forming capitalism. Institutional change in the German political economy, Oxford

Thelen, K. (2012): Varieties of capitalism: trajectories of liberalization and the new politics of social solidarity, in: Annual Review of Political Science 15 (1), S. $137-159$

\section{AUTOREN}

JOCHEN CLASEN ist Professor für Vergleichende Sozialpolitik an der University of Edinburgh. Arbeitsschwerpunkte: Sozialpolitik im Ländervergleich, Reform der Sozialsysteme, Arbeitsmarktpolitik.

jochen.clasen@ed.ac.uk

DANIEL CLEGG ist Senior Lecturer für Sozialpolitik an der University of Edinburgh. Arbeitsschwerpunkte: Vergleichende Sozialpolitik, Soziale Sicherung, Arbeitsmarktpolitik.

daniel.clegg@ed.ac.uk 